\title{
A supercritical superfluid and vortex unbinding following a quantum quench
}

\author{
L. Mathey ${ }^{1}$ and A. Polkovnikov ${ }^{2}$ \\ ${ }^{1}$ Joint Quantum Institute, National Institute of Standards and Technology and University of Maryland, Gaithersburg, MD 20899 \\ ${ }^{2}$ Department of Physics, Boston University, 590 Commonwealth Ave., Boston, MA 02215
}

(Dated: October 29, 2018)

\begin{abstract}
We study the dynamics of the relative phase of a bilayer of two-dimensional superfluids after the two superfluids have been decoupled, using truncated Wigner approximation. On short time scales the relative phase shows "light cone" like thermalization and creates a metastable superfluid state, which can be supercritical. On longer time scales this state relaxes to a disordered state due to dynamical vortex unbinding. This scenario of dynamically suppressed vortex proliferation constitutes a reverse-Kibble-Zurek effect. We observe dynamics of creation of vortex anti-vortex pairs and their consequent motion. Our predictions can be directly measured in interference experiments [1].
\end{abstract}

PACS numbers:

The understanding of order is one of the main objectives of many-body theory. Apart from topological order and exotic order 2], most phases can be characterized through the long-range behavior of their correlation functions. For example, a system of a single species of bosons in two spatial dimensions at finite temperature shows two different regimes: at low temperatures the correlation function of the boson operator $b(x)$ decays as a powerlaw $G(x) \equiv\left\langle b(x)^{\dagger} b(0)\right\rangle \sim|x|^{-\tau / 4}$, with a scaling exponent $\tau$, at higher temperatures it decays exponentially $\left\langle b(x)^{\dagger} b(0)\right\rangle \sim \exp \left(-|x| / x_{0}\right)$, with some coherence length $x_{0}$. The transition between these two regimes, occurring at the critical exponent $\tau_{c}=1$, is the famous BerezinskyKosterlitz-Thouless (BKT) transition [3]. It has been recently observed in ultra-cold atom systems [1, 4].

The power-law scaling of the quasi-superfluid state is due to thermally excited phonons. The gapless spectrum and the low dimension of the system lead to a fluctuating phase at all finite temperatures. If these were the only excitations of importance, the system would show powerlaw scaling at any temperature with the exponent $\tau$ being proportional to the temperature $T$ (see e.g. [5]). However, vortices can drive the system to a disordered state, in which the correlation function decays exponentially. In the quasi-superfluid phase vortex-antivortex (V-AV) pairs lead only to a small renormalization of the scaling exponent. These V-AV pairs are 'confined', i.e., they do not separate spatially significantly. However, above the transition temperature, due to entropic effects and due to screening, these excitations become 'deconfined', i.e., vortices and antivortices can separate spatially.

In this paper we study the dynamics of such a system, following a fast ramp across the transition, coming from the ordered side. We imagine two $2 \mathrm{D}$ superfluids that are coupled by a tunneling energy $J_{\perp}$, which leads to a phase-locked superfluid (SF) state, in which the fluctuations of the relative phase are suppressed, while the total phase correlations show algebraic scaling [6]. The critical temperature of this state typically lies well above the BKT temperature $T_{c}$ of the uncoupled system. We

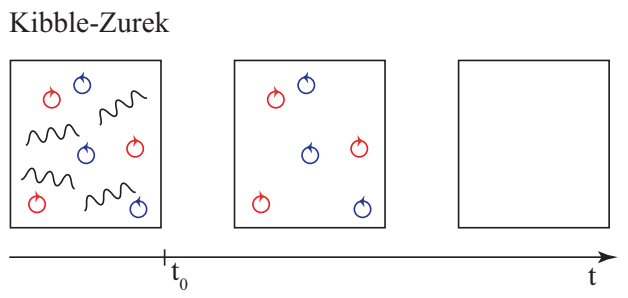

reverse Kibble-Zurek

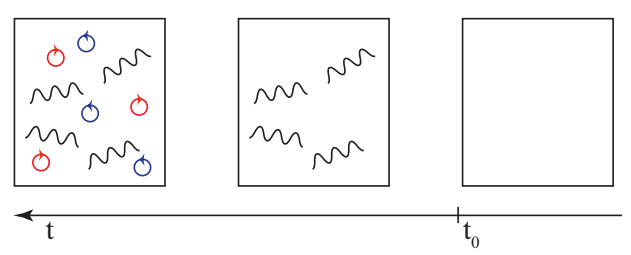

FIG. 1: Illustration of the Kibble-Zurek mechanism, which describes ramping across a phase transition from the disordered phase, and its counterpart, the reverse-Kibble-Zurek mechanism. The defining feature of the latter is the dynamical suppression of vortex unbinding, which happens long after phonons reach steady quasi-equilibrium state.

ask the question how the system transitions to its new equilibrium state after the two SFs have been decoupled by turning off $J_{\perp}$. (The case of $1 \mathrm{D}$ SFs has been studied in Refs. $7,[8,[9,10]$.) We find that on short time scales vortices are not important and the system shows 'lightcone'-like behavior in accord with prediction of Ref. 11] for 1D systems. Qualitatively this behavior comes from the fact that at distances larger than the product of a characteristic velocity and the time the correlation functions are not causally connected and thus decay in time but do not depend on the distance. Conversely at distances smaller than this product the correlation functions freeze in time and depend only on distance between the two points.

The state that emerges after this vortex free evolution is a metastable SF state. The correlation function shows algebraic scaling with some exponent that can be related to the initial temperature and the coupling energy of the 
initial state. However, the exponent of that state can be supercritical, that is, the correlation function can fall off faster than $|x|^{-1 / 4}$. This state can not exist in equilibrium and can be thought of as a 'superheated' superfluid.

On longer time scales this metastable state will relax to thermal equilibrium phase. If the effective temperature of the metastable superfluid is small then vortices do not unbind and thus the thermal equilibrium remains superfluid. If the metastable state is overheated then the relaxation to equilibrium is accomplished by vortex unbinding and the system becomes thermal Bose gas.

In Fig. 1] we illustrate this process, and contrast it to the Kibble-Zurek (KZ) mechanism [12, 13]. The latter refers to the case of ramping across a phase transition, from the disordered side. For a 2D superfluid that would be the thermal Bose gas phase, in which vortices are deconfined. If the system is then ramped across the transition with a fast quench, some of these vortices can survive on a very long time scale, their recombination with antivortices is suppressed (upper part of Fig. 11). The case that we consider here is ramping across the transition from the ordered side. The coupling $J_{\perp}$ between the two layers suppresses both phonons and vortices. When the system is decoupled phonons propagate very fast creating a metastable quasi-equilibrium state (second panel of the lower part of Fig. 1). Only much later equilibration of vortices occur, leading to thermal equilibrium. This clear separation of time scales allows us to refer to this process as the reverse-Kibble-Zurek (rKZ) mechanism.

We emphasize that despite the BKT transition is driven by thermal fluctuations, the mechanism of vortex or phonon creation in the process we consider can be triggered by initial quantum fluctuations. Indeed when the superfluids are strongly coupled together and the initial temperature is small the density (the momentum conjugate to the phase) has large fluctuations because of the zero point motion. The heating mechanism of this system can be thought of as enhancement of this zero point motion following the quench. This enhancement of fluctuations can not be captured within conventional mean-field approaches.

To model the relative phase dynamics we consider an $\mathrm{XY}$-model [6] and include a hopping term

$$
\begin{aligned}
H= & \Omega_{0}\left(-\sum_{<i j>} \frac{\kappa}{\pi} \cos \left(\phi_{i}-\phi_{j}\right)+\frac{\pi}{2 \kappa} \sum_{i} n_{i}^{2}\right. \\
& \left.-V(t) \sum_{i} \cos \left(\sqrt{2} \phi_{i}\right)\right)
\end{aligned}
$$

where $\Omega_{0}$ is an overall (Josephson) energy scale, $\kappa$ describes the ratio of kinetic and potential energies. We can formally replace these parameters by $\Omega_{0} \kappa / \pi=2 J n$, $\pi \Omega_{0} / \kappa=U$ (so that $\Omega_{0}=\sqrt{2 \operatorname{JnU}}, \kappa=\pi \sqrt{2 \operatorname{Jn} / U}$ ) and $V(t)=2 J_{\perp}(t) n / \Omega_{0}$, which gives a coarse-grained representation of the Hubbard model, where the Bose operators have been written in a phase-density represen- tation. In the Hubbard model $J$ is the in-plane hopping, $U$ is the interaction energy, $n$ is the average number of bosons per site, and $J_{\perp}$ is an inter-layer hopping. In the continuum limit the lattice size is approximately given by the healing length in the system.

It is convenient to introduce the rescaled quantities $\tilde{t}=\Omega_{0} t / \hbar, \tilde{\phi}=\sqrt{\frac{\kappa}{\pi}} \phi$, and $\tilde{n}=\sqrt{\frac{\pi}{\kappa}} n$. In terms of these, the equations of motion (EOMs) are

$$
\begin{aligned}
\frac{d \tilde{\phi}_{i}}{d \tilde{t}} & =-\tilde{n}_{i} \\
\frac{d \tilde{n}_{i}}{d \tilde{t}} & =-\frac{\sqrt{2}}{\beta} \sum_{j_{i}} \sin \left(\frac{\beta\left(\tilde{\phi}_{j_{i}}-\tilde{\phi}_{i}\right)}{\sqrt{2}}\right)+V(t) \beta \sin \beta \tilde{\phi}_{i}(2)
\end{aligned}
$$

where we defined $\beta=\sqrt{2 \pi / \kappa}$. The indices $j_{i}$ describe the four neighboring sites of site $i$.

We model the relative phase using a numerical implementation of the truncated Wigner approximation (TWA) (see Refs. 14 for a review): The expectation of any quantity at some time $t>0$ can be determined by sampling over a Wigner distribution at time $t=0$, and solving the classical equations of motion from 0 to $t$. This approximation is accurate either at short times, or with nearly harmonic systems [15]. In our case initial "light-cone" stage of dynamics is well described within the quadratic Bogoliubov theory where TWA is exact. At longer times, when nonlinear dynamics takes over, we expect TWA to remain applicable because by that time relevant momentum modes become highly occupied justifying the validity of the approach [14]. The advantage of this method is that each run strongly resembles a single realization of experiment, especially for a positively defined initial Wigner function. Thus to a very good precision one can mimic actual experiments. In Fig. 2 we plot time evolution of the relative phase for a single realization on initial conditions. One can observe V-AV pair formation at short times and their consequent unbinding.

We can calculate the Wigner distribution at $t=0$, assuming that $J_{\perp}$ is larger than the other energy scales. In this limit the phase fluctuations can be described within the Bogoliubov approximation, so the system reduces to a sum of oscillators. The Fourier modes $\tilde{\phi}_{q}$ and $\tilde{n}_{q}$ at $t=0$ are distributed according to (see Ref. [16])

$$
W \sim \exp \left(-\frac{\tilde{\phi}_{q}^{2}}{2 \sigma_{q}^{2} r_{q}}-\frac{2 \sigma_{q}^{2} \tilde{n}_{q}^{2}}{r_{q}}\right)
$$

with $\sigma=1 / \sqrt{2 \omega_{q}}, r_{q}=1 / \tanh \left(\omega_{q} / 2 T_{0}\right)$, and $\omega_{q}=$ $\sqrt{4 \sin \left(q_{x} / 2\right)^{2}+4 \sin \left(q_{y} / 2\right)^{2}+V \beta^{2}}, T_{0}$ being initial temperature. We use this method to extract the equal time correlation function:

$$
G(x, t)=\left\langle\exp \left[i \sqrt{2} \phi_{j}(t)-i \sqrt{2} \phi_{j+x}(t)\right]\right\rangle,
$$

where $x$ is an integer separation between the points and $t$ is the time after decoupling (see Fig. 3). Because we 

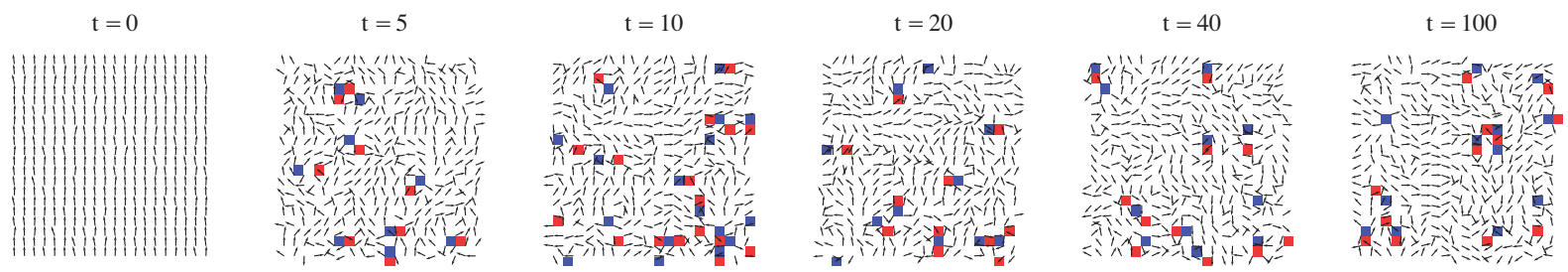

FIG. 2: Temporal evolution of the relative phase for a single realization of initial conditions. The parameters of the systems are $V=100, \kappa=10$ and $T_{0}=2$. Vortices are marked red, anti-vortices blue.
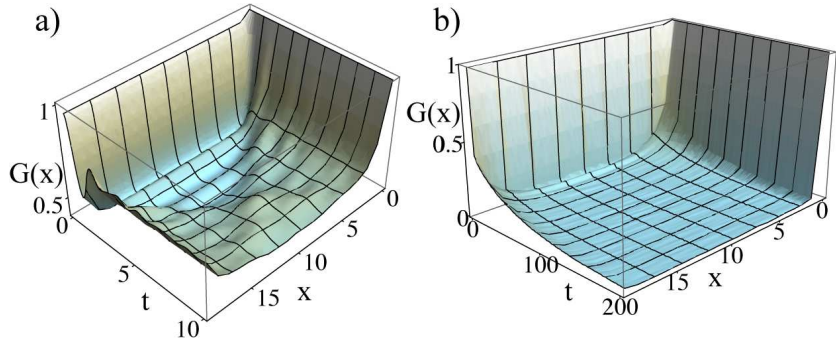

FIG. 3: Plot of short (left) and long (right) time behavior of the correlation function as a function of time and space. The parameters a $T_{0}=3$, for $\kappa=10$ and $V=20$ for the left graph and $T_{0}=1, \kappa=8$, and $V=80$ for the right graph. At short times the dynamics separates into instantaneous, damped oscillatory behavior, and a 'light cone' like pulse forming metastable quasi-superfluid state. At longer time scales the correlation function shows exponential decay due to dynamical vortex unbinding.

are using periodic boundary conditions $G(x, t)$ depends only on the separation between the points $x$. Note that this correlation function (or rather $\int_{0}^{x} d x^{\prime} G\left(x^{\prime}, t\right)$ ) can be directly measured in interference experiments [1, 7, 17]. We indeed see very clear emergence of the light cone thermalization: At separations larger than $2 v t$, where $v$ is characteristic phonon velocity, $G(x, t)$ is almost $x$ independent - it uniformly decreases in time. Once $2 v t>x$ the correlations freeze in time and depend only on $x$. We find that the state that emerges within the light cone shows algebraic scaling, and therefore can be referred to as a quasi-superfluid. At much longer times scale the correlation function clearly relaxes to the exponential equilibrium shape due to vortex unbinding.

Due to the relation between the power-law exponent and temperature in equilibrium, we can associate an effective temperature $T^{\star}$ with the state inside the light cone. We can estimate this effective temperature by considering the linearized dynamics, which neglects vortices. Then Eqs. (2) have the solution:

$$
\begin{aligned}
& \tilde{\phi}_{k}(t)=\tilde{\phi}_{k}(0) \cos \omega_{k} t-\tilde{n}_{k}(0) / \omega_{k} \sin \omega_{k} t \\
& \tilde{n}_{k}(t)=\tilde{\phi}_{k}(0) \omega_{k} \sin \omega_{k} t+\tilde{n}_{k}(0) \cos \omega_{k} t .
\end{aligned}
$$

We then consider the quadrature

$$
\left\langle\phi_{k}^{2}(t)\right\rangle=\frac{r_{k, 0}}{2 \omega_{k, 0}} \cos ^{2}\left(\omega_{k} t\right)+\frac{r_{k, 0} \omega_{k, 0}}{2 \omega_{k}^{2}} \sin ^{2}\left(\omega_{k} t\right) .
$$
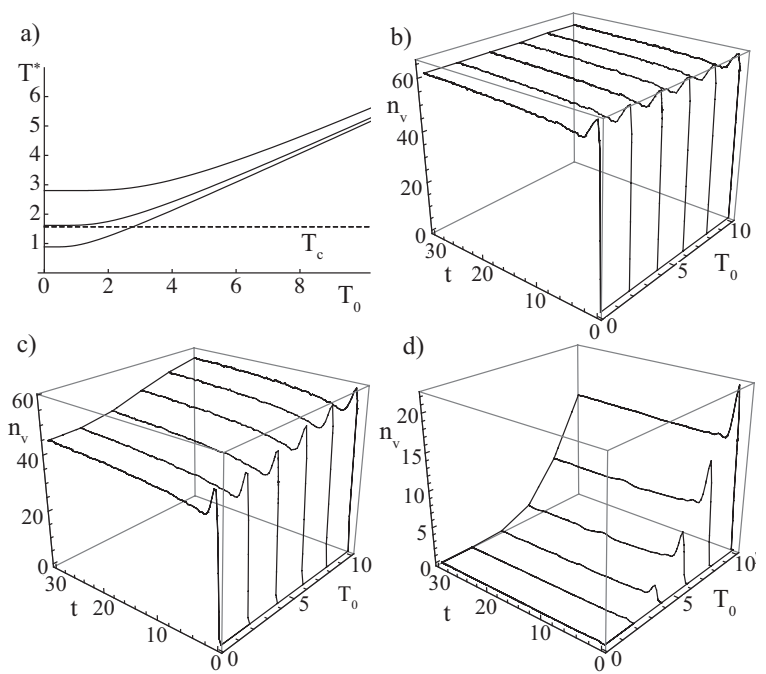

FIG. 4: a) $T^{\star}$, as given in Eq. 8 for $\kappa=1,3,10$, from top to bottom, and for $V=20$. The line $T_{c}=\pi / 2$ is the BKT temperature of the uncoupled SFs. b) - d) Simulations for these values of $\kappa$ and $V$.

where $r_{k, 0}$ and $\omega_{k, 0}$ correspond to the quantities at $t=0$, that is to $V(0)=V$, and $\omega_{k}$ to the dispersion with $V=0$. The long-time limit $\left\langle\phi_{k}^{2}(t \rightarrow \infty)\right\rangle$ is $r_{k, 0} / 4 \omega_{k, 0}+$ $r_{k, 0} \omega_{k, 0} / 4 \omega_{k}^{2}$. We equate this formally to a thermal ensemble of momentum-dependent 'temperature' $T_{k}^{\star}$, i.e. $\left\langle\phi_{k}^{2}(t \rightarrow \infty)\right\rangle=r_{k}^{\star} / \omega_{k}$, where $r_{k}^{\star}=1 / \tanh \left(\omega_{k} / 2 T_{k}^{\star}\right)$. We solve for $T_{k}^{\star}$, and find that for large $V \beta^{2}$ it simplifies to a single value, independent of $k$ :

$$
T^{\star}=\frac{\sqrt{V \beta^{2}}}{4 \tanh \left(\sqrt{V \beta^{2}} / 2 T_{0}\right)} .
$$

For small $T_{0}$ we have $T^{\star} \approx \frac{\sqrt{V \beta^{2}}}{4}\left(T^{\star}=2 J_{\perp} / J\right.$ in terms of original Hubbard parameters), that is, the temperature is fully determined by the initial coupling energy. The coupling energy between the two layers is transferred into the in-plane kinetic energy. We note that if $J_{\perp}$ exceeds the chemical potential $U n$ the quantum rotor model becomes inadequate and then $T^{\star}$ saturates at the value $T^{\star} \sim U n / J$. For large $T_{0}$ we have $T^{\star} \approx \frac{T_{0}}{2}$. This is a reflection of the doubling of the degrees of freedom when two layers are uncoupled. In Fig. 4 a) we plot $T^{\star}$ for 


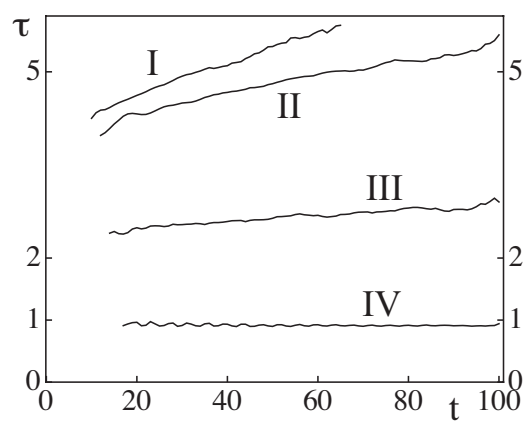

FIG. 5: Exponent $\tau$ extracted from fitting the correlation function $G(x)$ to the algebraic form, for different initial couplings. We use $T_{0}=1$ and $\kappa=8$, and $V=80,70,50$, and 20, corresponding to curves I to IV. Except for the curve I corresponding to Fig. 3-b) the correlation function can be fitted with an algebraic function throughout the time interval shown here.

$V=20$ and for different value of $\kappa$. For $\kappa=1$ corresponding to large $J_{\perp}, T^{\star}$ is always above the critical temperature $T_{c}=\pi / 2$, for $\kappa=10$, it crosses it as initial temperature increases. We therefore expect to see very little vortex formation for small initial temperatures for $\kappa=10$, and many vortices for all temperatures for $\kappa=1$. This is indeed the case as we show in Fig. 4, which plots the number of vortices $n_{v}$ versus time and initial temperature for three different values of $\kappa$. This number is obtained by counting the plaquettes with a phase winding of $2 \pi$, and then by averaging over many runs.

To quantify the crossover from the supercritical superfluid state to the normal phase we fit the correlation function $G(x, t)$ at different times numerically using algebraic $\left(G(x, t)=c(L / \pi|\sin (\pi x / L)|)^{-\tau / 4}\right)$ and an exponential $(G(x, t)=c \exp (-|\sin (\pi x / L)| / a))$ functions. In equilibrium the algebraic exponent $\tau$ would be the relative temperature $T / T_{c}$. Any value above 1 is therefore supercritical. We use these two fitting functions in four examples with $T_{0}=1$ and $\kappa=8$, but with different initial couplings $V=80,70,60,20$. In Fig. 5 we show the exponent $\tau$ as a function of time for these cases. In all cases $G(x, t)$ develops algebraic scaling after the light-cone dynamics. Note that for large initial couplings (I-III) the emerging scaling exponent $\tau$ is well above the critical exponent. At longer times the exponent slowly increases in time. During this process, the correlation function can still be well fitted with an algebraic function. Eventually the correlation function develops exponential scaling. This regime is reached for $V=80$ (I) within the time interval shown in Fig. [5 signalling that the thermal Bose gas phase has been reached due to vortex unbinding. For $V=70$ (II) and $V=50$ (III) the time scale of vortex unbinding is longer then the time interval shown. For $V=20$ (IV) the system remains in a quasi-superfluid state. We conclude from these examples that the supercritical superfluid has a long life-time and the exponent $\tau$ can significantly ex- ceed the maximum equilibrium value. Such metastable states should be thus experimentally feasible.

In conclusion, we have studied the dynamics of the relative phase of a bilayer of superfluids in 2D, after the hopping between them has been turned off rapidly. We find that on short time scales the dynamics of the correlation function shows a "light-cone"-like behavior. The system then develops an algebraic phase that can be stable or metastable. The latter can be thought of as a superheated superfluid. On long time scales this metastable superfluid relaxes to a disordered state via creating of vortex-antivortex pairs and their consequent unbinding.

Our predictions can be directly probed in experiment. The behavior of the relative of phase of two superfluids can be studied by interference experiments [17], so both the supercritical state and the algebraic-to-exponential evolution can be tested. Vortex unbinding can also be demonstrated by direct observation of free vortices [1]. The time scale of the light-cone dynamics can be estimated as $t_{L C} \sim L / v$, where the system size $L \sim 10^{-4} \mathrm{~m}$ and the phonon velocity $v \sim 10^{-3} \mathrm{~m} / \mathrm{s}$ leads to $t_{L C} \sim$ $0.1 \mathrm{~s}$. The rate of vortex unbinding is exponentially suppressed compared to the BKT energy scale [18].

The authors acknowledge useful discussions with A.H. Castro Neto and E. Altman. A.P. was supported by AFOSR YIP and Sloan Foundation. L.M. acknowledges support from NRC/NIST, NSF Physics Frontier Grant PHY-0822671 and Boston University visitor's program.

[1] Z. Hadzibabic, et al., Nature 441, 1118 (2006).

[2] X.-G. Wen, Quantum Field Theory of Many-Body Systems (Oxford University Press, Oxford, 2004).

[3] J. M. Kosterlitz and D. J. Thouless, J. Phys. C 6, 1181 (1973); V. S. Berezinskii, JETP 34, 610 (1972).

[4] P. Clade, et al., cond-mat/0805.3519.

[5] P. M. Chaikin and T. C. Lubensky, Principles of Condensed Matter Physics (Cambridge University Press, Cambridge, 1995).

[6] L. Mathey, et al., EuroPhys. Lett. 81, 10008 (2008); M. A. Cazalilla, et al., Phys. Rev. A 75, 051603(R) (2007).

[7] S. Hofferberth, et al., Nature 449, 324 (2007).

[8] A. A. Burkov, et al., Phys. Rev. Lett. 98, 200404 (2007).

[9] R. Bistritzer and E. Altman, PNAS 104, 9955 (2007).

[10] I. E. Mazets, J. Schmiedmayer, arXiv:0806.4431

[11] P. Calabrese and J. Cardy, Phys. Rev. Lett. 96, 136801 (2006); J. Stat. Mech. - Theor. and Exp., P06008 (2007).

[12] T. W. B Kibble, J. Phys. A 9, 1387 (1976); Physics Today 60, 47 (20007).

[13] W. H. Zurek, Nature 317, 505 (1985).

[14] P. B. Blakie, et al., Advances in Physics 57, 363 (2008).

[15] A. Polkovnikov, Phys. Rev. A 68, 053604 (2003); ibid arXiv:0905.3384

[16] A. Polkovnikov, V. Gritsev, Nature Phys. 4, 477 (2008).

[17] A. Polkovnikov, E. Altman, and E. Demler, Proc. Natl. Acad of Sci. USA 103, 6125 (2006).

[18] in preparation. 